\title{
A CLASS OF GENERALIZED SUPERSOLUBLE GROUPS
}

\author{
Adolfo Ballester-Bolinches and Tatiana Pedraza
}

\begin{abstract}
This paper is devoted to the study of groups $G$ in the universe $c \overline{\mathfrak{L}}$ of all radical locally finite groups with min- $p$ for all primes $p$ such that every $\delta$-chief factor of $G$ is either a cyclic group of prime order or a quasicyclic group. We show that within the universe $c \overline{\mathfrak{L}}$ this class of groups behaves very much as the class of finite supersoluble groups.
\end{abstract}

\section{Introduction}

A group $G$ is said to be supersoluble if it has a finite normal series of cyclic factors. A chief factor of a supersoluble group is cyclic of prime order and a maximal subgroup has prime index. In fact these two properties characterize finite supersoluble groups. It is well known that in the universe of all finite groups the class of supersoluble groups forms a subgroup-closed saturated formation which is intermediate between the classes of nilpotent groups and soluble groups. Several authors have investigated supersoluble groups, not necessarily finite, and generalizations (hypercyclic groups and locally supersoluble groups) extending some results of finite supersoluble groups and establishing connections between these classes of generalized supersoluble groups.

In this paper, we introduce a class of generalized supersoluble groups in the universe $c \overline{\mathfrak{L}}$ of all radical locally finite groups with min- $p$ for all primes $p$ : the class $\mathcal{U}^{*}$. It is intermediate between the classes of supersoluble $c \overline{\mathfrak{L}}$-groups and hypercyclic $c \overline{\mathfrak{L}}$-groups and it plays a similar role in the class $c \overline{\mathfrak{L}}$ as supersoluble groups do in the class of all finite groups.

2000 Mathematics Subject Classification. 20F16, 20F19, 20 F50.

Key words. Locally finite groups, generalized supersoluble groups, major subgroups. This research is supported by Grant BFM-2001-1667-C03-03, МСyT (Spain) and FEDER (European Union). 


\section{Preliminaries}

The main purpose of this section is to establish the notation, terminology and some results which will be used throughout this paper. Notation that is not specifically cited here is consistent with that used in $[\mathbf{7}],[\mathbf{8}]$ and $[\mathbf{1 0}]$.

An infinite group can have insufficient maximal subgroups or even none at all. In order to avoid this situation, Tomkinson [11] introduces the notion of major subgroup. We recall this definition. Let $U$ be a subgroup of a group $G$ and consider the properly ascending chains

$$
U=U_{0}<U_{1}<\cdots<U_{\alpha}=G
$$

from $U$ to $G$, then $m(U)$ is the least upper bound of the types $\alpha$ of all such chains. Clearly $m(U)=1$ if and only if $U$ is a maximal subgroup of $G$.

A proper subgroup $M$ of $G$ is said to be a major subgroup of $G$ if $m(U)=m(M)$ whenever $M \leq U<G$. This is a nice extension of the concept of maximal subgroup in the sense that every proper subgroup of a group $G$ is always contained in a major subgroup of $G[\mathbf{1 1},(2.3)]$. In particular, the intersection of all major subgroups of a group $G$, denoted by $\mu(G)$, is a proper subgroup of $G$ with properties similar to those of the Frattini subgroup of a finite group.

In the sequel, we tacitly assume that all groups belong to the class c $\overline{\mathfrak{L}}$ of all radical locally finite groups with min-p for all primes $p$.

Let $G$ be a group and let $M$ be a major subgroup of $G$. Denote $M_{G}=\operatorname{Core}_{G}(M)$. Then $G / M_{G}$ is either a finite soluble primitive group, if $M$ is a maximal subgroup of $G$, or a semiprimitive group, if $M$ is not maximal in $G$ (here, a group $G$ is said to be semiprimitive if it is the split extension, $G=[D] M$, of a faithful divisibly irreducible $\mathbb{Z} M$-module $D$ by a finite soluble group $M$ ). This result, proved in [2], confirms the importance of major subgroups in the study of the structure of groups and motivates some definitions which are in some sense extensions of well known ones in the finite universe.

Definition 1. Suppose that $G$ is a group and let $M$ be a major subgroup of $G$. We define

$$
D_{M} / M_{G}= \begin{cases}\operatorname{Soc}\left(G / M_{G}\right), & \text { if } M \text { is a maximal subgroup of } G \\ \left(G / M_{G}\right)^{0}, & \text { if } M \text { is not a maximal subgroup of } G .\end{cases}
$$


In both cases, $D_{M} / M_{G}=F\left(G / M_{G}\right),\left(D_{M} / M_{G}\right) \cap\left(M / M_{G}\right)=1$ and $\mathrm{C}_{G / M_{G}}\left(D_{M} / M_{G}\right)=D_{M} / M_{G}$ for every major subgroup $M$ of $G$.

Let $G$ be a group and consider two normal subgroups $H$ and $K$ of $G$ such that $K$ is contained in $H$. Then $H / K$ is called a $\delta$-chief factor of $G$ if $H / K$ is either a minimal normal subgroup of $G / K$ or a divisibly irreducible $\mathbb{Z} G$-module, that is, $H / K$ has no proper infinite $G$-invariant subgroups. Every $\delta$-chief factor is either an elementary abelian finite $p$-group for some prime $p$ or a direct product of finitely many quasicyclic $p$-groups for some prime $p$ (see $[\mathbf{7},(1.2 .4)])$.

Let $G$ be a group. We say that $G$ is a $\mathcal{U}^{*}$-group if every $\delta$-chief factor of $G$ is either a cyclic group of prime order or a quasicyclic group. Obviously the class $\mathcal{U}^{*}$ is a class of generalized supersoluble groups in the universe $c \overline{\mathfrak{L}}$ because every finite $\mathcal{U}^{*}$-group is supersoluble and every supersoluble $c \overline{\mathfrak{L}}$-group is finite and so it is a $\mathcal{U}^{*}$-group.

On the other hand, since every group contains minimal normal subgroups it follows that every $\mathcal{U}^{*}$-group is hypercyclic (and hence locally supersoluble [1]). Moreover the class $\mathcal{U}^{*}$ is intermediate between the classes of supersoluble groups and hypercyclic groups. On one hand, every quasicyclic $p$-group, $p$ a prime, is a non-supersoluble $\mathcal{U}^{*}$-group. On the other hand, let $G$ be a cyclic group of order $2^{2}$. Consider a divisibly irreducible $\mathbb{Z} G$-module $A$, faithful for $G$, such that $A$ is a periodic 2-group ( $A$ always exists and it is unique up to isomorphism by $[\mathbf{9}$, (3.5)]). Then, applying $[\mathbf{9},(5.9)], A$ has rank 2. In particular, the Chernikov group $X=A G$ is not in the class $\mathcal{U}^{*}$. Moreover, if $K$ is a normal subgroup of $X$ such that $X / K \neq 1$ then $X / K$ contains a minimal normal subgroup $N / K$. Since $X / K$ is a 2 -group then it is locally nilpotent and then, by $[\mathbf{7},(1.2 .6)], N / K$ is a cyclic group of order 2 . We conclude that $X$ is a hypercyclic group.

Let $\mathfrak{B}$ be the class of all groups in which every proper subgroup has a proper normal closure. The class $\mathfrak{B}$ has been introduced and studied in [3], [4]. The results of these papers show that this class is intermediate between nilpotent and locally nilpotent groups, and that it is the natural generalization of the class of finite nilpotent groups from the finite universe to the universe $c \overline{\mathfrak{L}}$. We show that the largest normal $\mathfrak{B}$-subgroup of a group is the Fitting subgroup and every $\delta$-chief factor of a group $G$ in $\mathfrak{B}$ is central in $G$. Consequently, every $\delta$-chief factor of $G$ has rank one and $G$ is a $\mathcal{U}^{*}$-group. Therefore, we obtain in the universe $c \overline{\mathfrak{L}}$ similar inclusions to the finite universe for these classes of generalized nilpotent 
groups and generalized supersoluble groups:

$$
\{\text { Abelian groups }\} \subset\{\text { Nilpotent groups }\} \subset \mathfrak{B} \subset \mathcal{U}^{*}
$$

and all these inclusions are proper.

Let $p$ be a prime. We say that a group $G$ is a $\mathfrak{B}_{p}$-group if $G$ is $p$-nilpotent and the Sylow $p$-subgroups of $G$ are nilpotent. The class $\mathfrak{B}_{p}$ is a local version of the class $\mathfrak{B}$. The results of the paper [5] show that $\mathfrak{B}_{p}$ is a subgroup-closed formation which plays the same role in the universe $c \overline{\mathfrak{L}}$ as finite $p$-nilpotent groups do in the finite one. In particular, every group $G$ has a unique largest normal $\mathfrak{B}_{p^{-}}$subgroup denoted by $\delta_{p^{\prime} p}(G)$, for every prime $p$, which is the intersection of the centralizers of all $\delta$-chief factors of $G$ which are $p$-groups and $F(G)=\bigcap_{p} \delta_{p^{\prime} p}(G)$.

Let $G$ be a $\mathcal{U}^{*}$-group and let $p$ be a prime. If $H / K$ is a $\delta$-chief factor of $G$ which is a $p$-group then $G / \mathrm{C}_{G}(H / K)$ is a cyclic group of order dividing $p-1$ if $p \neq 2$ or a cyclic group of order dividing 2 if $p=2$ by [7, (1.5.18), (1.5.19)]. Therefore $G / \delta_{p^{\prime} p}(G)$ is in the class $\mathfrak{A}(p-1)$ if $p \neq 2$ or in the class $\mathfrak{A}(2)$ if $p=2$, where $\mathfrak{A}(n)$ denotes the class of all abelian groups with exponent dividing $n$. Moreover $G / O_{p^{\prime} p}(G) \in \mathfrak{A}(p-1)$ for every prime $p$ because $O_{p^{\prime} p}(G)$ is the intersection of the centralizers of all $p$-chief factors of $G$ by $[\mathbf{7},(6.2 .4)]$.

\section{The results}

A finite group $G$ is supersoluble if and only if for every prime $p$, $G / O_{p^{\prime} p}(G)$ is abelian of exponent dividing $p-1$. If $G$ is a $c \overline{\mathfrak{L}}$-group, that condition does not imply that $G \in \mathcal{U}^{*}$ (consider for instance the example of a 2 -group in Section 2 which is not a $\mathcal{U}^{*}$-group). Our first result provides a necessary and sufficient condition for a group $G$ to be a $\mathcal{U}^{*}$-group.

Theorem 1. A group $G$ is a $\mathcal{U}^{*}$-group if and only if for every prime $p$, $G / \delta_{p^{\prime} p}(G)$ is in the class $\mathfrak{A}(p-1)$ if $p \neq 2$ or in the class $\mathfrak{A}(2)$ if $p=2$, where $\mathfrak{A}(n)$ denotes the class of all abelian groups with exponent dividing $n$.

Proof: Let $G$ be a group such that for every prime $p, G / \delta_{p^{\prime} p}(G)$ is in the class $\mathfrak{A}(p-1)$ if $p \neq 2$ or in the class $\mathfrak{A}(2)$ if $p=2$. Consider $H / K$ a chief factor of $G$. In particular $H / K$ is a $p$-group for some prime $p$. Since $G / \mathrm{C}_{G}(H / K)$ is in the class $\mathfrak{A}(p-1)$ if $p \neq 2$ or in the class $\mathfrak{A}(2)$ if $p=2$ it follows from [8, B, (9.8)] that $H / K$ is a cyclic group of order $p$. Suppose now that $H / K$ is a divisibly irreducible $\mathbb{Z} G$-module. In particular $H / K$ is a $p$-group for some prime $p$. Suppose that $p \neq 2$. 
Then $L=G / \mathrm{C}_{G}(H / K)$ is in the class $\mathfrak{A}(p-1)$. Since $H / K$ is a divisibly irreducible $\mathbb{Z} L$-module which is faithful for $L$ it follows from $[\mathbf{9},(3.1)]$ that $L$ is a cyclic group. Since $|L|$ divides $p-1$, it follows from $[\mathbf{9},(3.4)]$ that $H / K$ has rank 1 , that is, $H / K$ is a quasicyclic $p$-group. Assume now that $p=2$. Then $L=G / \mathrm{C}_{G}(H / K)$ is in the class $\mathfrak{A}(2)$. Applying [9, (3.1)] we have that $L$ is trivial or a cyclic group of order 2 . Consequently $H / K$ has rank 1 by $[\mathbf{9},(3.4)]$. We conclude that $G$ is a $\mathcal{U}^{*}$-group.

Our next results analyze the behaviour of $\mathcal{U}^{*}$ as a class of groups and they are motivated by the well known fact that, in the finite universe, the class of all supersoluble groups is a subgroup-closed saturated formation [8, VII, (2.19)].

Recall that a class $\mathfrak{F}$ of groups is said to be a formation if it satisfies the following properties:

1. If $G \in \mathfrak{F}$ and $N$ is a normal subgroup of $G$, then $G / N \in \mathfrak{F}$.

2. If $\left\{N_{i}\right\}_{i \in I}$ is a collection of normal subgroups of $G$ such that $G / N_{i} \in \mathfrak{F}$ for every $i \in I$ and $\bigcap_{i \in I} N_{i}=1$, then $G \in \mathfrak{F}$.

Theorem 2. The class $\mathcal{U}^{*}$ is a subgroup-closed formation.

Proof: It is clear that $\mathcal{U}^{*}$ is closed under taking epimorphic images, that is, $\mathcal{U}^{*}$ is $Q$-closed.

Let $\left\{N_{i}\right\}_{i \in I}$ be a collection of normal subgroups of a group $G$ such that $G / N_{i} \in \mathcal{U}^{*}$ for every $i \in I$ and $\bigcap_{i \in I} N_{i}=1$. Since $G / N_{i} \in \mathcal{U}^{*}$ we have that $G / N_{i} /\left(\delta_{p^{\prime} p}\left(G / N_{i}\right)\right)$ is in the class $\mathfrak{A}(p-1)$ if $p \neq 2$ or in the class $\mathfrak{A}(2)$ if $p=2$, for all $i \in I$. Let us denote by $G^{\mathfrak{A}(n)}$ the $\mathfrak{A}(n)$-residual of a group $G$. Suppose first that $p \neq 2$. Then $G^{\mathfrak{A}(p-1)} N_{i} / N_{i}=\left(G / N_{i}\right)^{\mathfrak{A}(p-1)} \leq \delta_{p^{\prime} p}\left(G / N_{i}\right)$ for all $i \in I$. In particular, $G^{\mathfrak{A}(p-1)} N_{i} / N_{i}$ is in the class $\mathfrak{B}_{p}$ for all $i \in I$. Since $\mathfrak{B}_{p}$ is a formation by [5, Theorem 1] we obtain that $G^{\mathfrak{A}(p-1)} \in \mathfrak{B}_{p}$. Consequently $G / \delta_{p^{\prime} p}(G) \in \mathfrak{A}(p-1)$. Suppose now that $p=2$. Then $G^{\mathfrak{A}(2)} N_{i} / N_{i}=\left(G / N_{i}\right)^{\mathfrak{A}(2)} \leq \delta_{2^{\prime} 2}\left(G / N_{i}\right)$ for all $i \in I$. In particular, $G^{\mathfrak{A}(2)} N_{i} / N_{i}$ is in the class $\mathfrak{B}_{2}$ for all $i \in I$. Therefore $G^{\mathfrak{A}(2)} \in \mathfrak{B}_{2}$ and hence $G / \delta_{2^{\prime} 2}(G) \in \mathfrak{A}(2)$. It follows from Theorem 1 that $G \in \mathcal{U}^{*}$. Consequently $\mathcal{U}^{*}$ is a formation.

We prove now that $\mathcal{U}^{*}$ is subgroup-closed. Let $G$ be a $\mathcal{U}^{*}$-group and let $H$ be a subgroup of $G$. We have to prove that $H$ is also a $\mathcal{U}^{*}$-group. We split the proof into three cases:

Case 1: $G$ is a Chernikov group such that $G^{0}$ (the radicable part of $G$ ) is a quasicyclic $p$-group for some prime $p$. Since $H^{0}$ is a divisible subgroup of $G^{0}$, then either $H^{0}=1$ or $H^{0}=G^{0}$. First we assume that $H^{0}=G^{0}$. 
Let $A / B$ be a $\delta$-chief factor of $H$. Since the class $\mathcal{U}^{*}$ is $Q$-closed, we may assume that $B=1$. If $A$ is a divisibly irreducible $\mathbb{Z} H$-module, then $A$ is a quasicyclic $p$-group. Suppose now that $A$ is a minimal normal subgroup of $H$. Then either $A \leq H^{0}$ or $A \cap H^{0}=1$. If $A$ is contained in $H^{0}$, then $A$ is a cyclic group of prime order $p$ because it is an elementary abelian. Assume that $A \cap H^{0}=1$. Since $A H^{0} / H^{0}$ is a minimal normal subgroup of $H / H^{0}$ and $H / H^{0}$ is a finite supersoluble group, it follows that $A H^{0} / H^{0}$ is a cyclic group of prime order and so is $A$. Suppose now that $H^{0}=1$, that is, $H$ is finite. If $A / B$ is a chief factor of $G$, then $A / B$ is a cyclic group of prime order. In particular $(H \cap A) /(H \cap B)$ is either trivial or a cyclic group of prime order. Hence a chief series of $G$ intersected with $H$ will yield, after deleting redundant terms, a chief series of $H$ (of finite length) with cyclic factors. Therefore $H \in \mathcal{U}^{*}$.

Case 2: $G$ is a Chernikov group with $O_{p^{\prime}}(G)=1$ for some prime $p$. Then $G=G^{0} M$, where $M$ is a finite subgroup of $G$. We can certainly assume that $G^{0} \neq 1$, since otherwise $G$ is a finite supersoluble group and so the result follows. Therefore $G^{0}$ is a divisible abelian $p$-group of finite rank. By $[\mathbf{1 2},(1.3)]$ there is a finite normal subgroup $C$ of $G$ contained in $G^{0}$ such that $G^{0} / C$ is a direct product of divisibly irreducible $\mathbb{Z} G$-modules, say

$$
G^{0} / C=\left(G_{1} / C\right) \times\left(G_{2} / C\right) \times \cdots \times\left(G_{n} / C\right) .
$$

Since $G_{i} / C$ is a $\delta$-chief factor of $G$ and $G \in \mathcal{U}^{*}$, we have that $G_{i} / C$ is a quasicyclic $p$-group for all $i \in\{1, \ldots, n\}$. Let us first assume that $C=1$ and denote $X_{1}=G_{2} \times G_{3} \times \cdots \times G_{n}$ and $X_{i}=G_{1} \times \cdots \times G_{i-1} \times G_{i+1} \times$ $\cdots \times G_{n}$ for all $i>1$. It is clear that $X_{i}$ is normal in $G$ for all $i$ and $\bigcap_{i \geq 1} X_{i}=1$. Moreover $G / X_{i} \cong G_{i} M$ and $\left(G_{i} M\right)^{0}=G_{i}$ is a quasicyclic $p$-group. Applying Case 1 , we have that $H /\left(H \cap X_{i}\right)$ is a $\mathcal{U}^{*}$-group for every $i$. Since $\mathcal{U}^{*}$ is a formation, we conclude that $H \in \mathcal{U}^{*}$. Assume now that $C \neq 1$. By the above argument, $H C / C$ is a $\mathcal{U}^{*}$-group. We prove that $H C \in \mathcal{U}^{*}$. Let $A / B$ be a $\delta$-chief factor of $H C$. There is no loss of generality in assuming that $B=1$. Suppose that $A$ is a minimal normal subgroup of $H C$. Then either $A \cap C=1$ or $A \cap C=A$. If $A \cap C=1$, it follows that $A C / C$ is a minimal normal subgroup of $H C / C \in \mathcal{U}^{*}$ and so $A$ is a cyclic group of prime order. Assume that $A \leq C$. Let

$$
1=C_{0} \triangleleft C_{1} \triangleleft C_{2} \triangleleft \cdots \triangleleft C_{s}=C
$$

be part of a chief series of $G$ passing through $C$, that is, $C_{i} / C_{i-1}$ is a minimal normal subgroup of $G / C_{i-1}$ for all $i \in\{1, \ldots, s\}$. Since $G \in \mathcal{U}^{*}$, we have that $C_{i} / C_{i-1}$ is a cyclic group of prime order for all $i$. On the other hand, since $1 \neq A \leq C$ there exists $j \in\{1, \ldots, s\}$ such that 
$A \leq C_{j}$ and $A \not \leq C_{j-1}$. In particular, $1 \neq A C_{j-1} / C_{j-1} \leq C_{j} / C_{j-1}$ and then $A \cong A C_{j-1} / C_{j-1}$ is a cyclic group of prime order. Suppose now that $A$ is a divisibly irreducible $\mathbb{Z}(H C)$-module. Then $A \cap C$ is a proper finite subgroup of $A$. This implies that $A /(A \cap C) \cong A C / C$ is a divisibly irreducible $\mathbb{Z}(H C / C)$-module and so it is a quasicyclic group. Consequently $A$ is a quasicyclic group. We have proved that $H C$ is a $\mathcal{U}^{*}$-group. Since $\mathcal{U}^{*}$ is a formation and $C$ is an abelian normal subgroup of $H C$, it follows from [2, Lemma 2] that $H \in \mathcal{U}^{*}$, which is our claim.

General case: By $[\mathbf{7},(2.5 .13)], G / O_{p^{\prime}}(G)$ is a Chernikov $\mathcal{U}^{*}$-group for every prime $p$. Applying Case 2, we have that $H /\left(H \cap O_{p^{\prime}}(G)\right)$ is a $\mathcal{U}^{*}$ group for every prime $p$. Since $\bigcap_{p}\left(H \cap O_{p^{\prime}}(G)\right)=1$ and $\mathcal{U}^{*}$ is a formation, we conclude that $H$ belongs to $\mathcal{U}^{*}$, which completes the proof.

Bearing in mind that the class of supersoluble groups in the finite universe is saturated, one can wonder if $\mathcal{U}^{*}$ could enjoy this property in our universe. The answer is negative as the following example shows:

Example 1. Let $X$ be the regular wreath product of a quasicyclic $p$-group and a cyclic group of order $p$, where $p$ is a prime number. Consider $G=X / \mathrm{Z}(X)$. Then $G=G^{0} M$ is a semiprimitive group such that $G^{0}$ is isomorphic to a direct product of $p-1$ quasicyclic $p$-groups and $M$ is a cyclic group of order $p$. Moreover, the group $G$ can be expressed as $G=\bigcup_{i>1} G_{i}$, where $G_{i}=\Omega_{i}\left(G^{0}\right) M$, for each natural number $i$ and $\left\{G_{i}: i \geq 1\right\}$ is an ascending chain of subgroups of $G$. Notice that $G_{i}$ is a finite $p$-group and hence a supersoluble group for each $i \geq 1$. However, if $p \neq 2, G$ is not a $\mathcal{U}^{*}$-group because $G^{0}$ is a divisibly irreducible $\mathbb{Z} G$-module which is not quasicyclic. Applying $[\mathbf{2}$, Theorem $\mathrm{A}]$, we have that $\mathcal{U}^{*}$ is not saturated.

Nevertheless, $\mathcal{U}^{*}$ is closed under taking extensions by Tomkinson's Frattini-like subgroup as our next result shows.

Theorem 3. Let $N$ be a normal subgroup of a group $G$ such that $N / \mu(G)$ is a $\mathcal{U}^{*}$-group. Then $N \in \mathcal{U}^{*}$.

Proof: Assume first that $G$ is a Chernikov group. Then $\mu(G)$ is finite by $[\mathbf{1 2},(1.2)]$. On the other hand, for every prime $p,(N / \mu(G)) / O_{p^{\prime} p}(N / \mu(G))$ is abelian of exponent dividing $p-1$ because $N / \mu(G) \in \mathcal{U}^{*}$. Moreover, the arguments used in $[\mathbf{1 1},(5.1)]$ allow us to show that $O_{p^{\prime} p}(N / \mu(G))=$ $O_{p^{\prime} p}(N) / \mu(G)$. Thus, for every prime $p, N / O_{p^{\prime} p}(N)$ is abelian of exponent dividing $p-1$.

Let $H / K$ be a $\delta$-chief factor of $N$. Then either $H \mu(G) / K \mu(G)$ is $N$-isomorphic to $H / K$ or $H \mu(G)=K \mu(G)$ and $H \cap \mu(G) / K \cap \mu(G)$ is 
$N$-isomorphic to $H / K$. Consequently, there is no loss of generality in assuming that either $K \leq H \leq \mu(G)$ or $\mu(G) \leq K \leq H$. In the first case, we have that $H / K$ is finite and therefore $H / K$ is a $p$-chief factor of $N$ for some prime $p$. In particular, $\operatorname{Aut}_{N}(H / K) \cong N / \mathrm{C}_{N}(H / K)$ is finite and abelian of exponent dividing $p-1$. Applying [8, $\mathrm{B},(9.8)], H / K$ is a cyclic group of order $p$. Assume now that $\mu(G) \leq K \leq H$. Then $H / K$ is isomorphic to a $\delta$-chief factor of $N / \mu(G) \in \mathcal{U}^{*}$ and consequently $H / K$ is a cyclic group of prime order or a quasicyclic group. We conclude that $N \in \mathcal{U}^{*}$.

In the general case, applying $[\mathbf{7},(2.5 .13)], G / O_{p^{\prime}}(G)$ is a Chernikov group for every prime $p$. Moreover, since $N / \mu(G) \in \mathcal{U}^{*}$ and $\mu(G) O_{p^{\prime}}(G) / O_{p^{\prime}}(G) \leq \mu\left(G / O_{p^{\prime}}(G)\right)$ it follows that $N O_{p^{\prime}}(G) / O_{p^{\prime}}(G)$ is a normal subgroup of $G / O_{p^{\prime}}(G)$ satisfying the hypothesis of the theorem. By the above argument, we obtain that $N /\left(N \cap O_{p^{\prime}}(G)\right)$ is a $\mathcal{U}^{*}$-group, for every prime $p$. Since $\mathcal{U}^{*}$ is a formation, we conclude that $N$ belongs to $\mathcal{U}^{*}$, as required.

Corollary 1. Let $G$ be a group. Then $G / \mu(G) \in \mathcal{U}^{*}$ if and only if $G \in \mathcal{U}^{*}$.

If $M$ is a major subgroup of a group $G$ such that $D_{M} / M_{G}$ is a cyclic group of prime order or a quasicyclic group then $G / M_{G}$ is a $\mathcal{U}^{*}$-group. This fact motivates the following:

Definition 2. Let $G$ be a group and let $M$ be a major subgroup of $G$. We define the extended index of $M$ in $G$, denoted by $q_{G}(M)$, as the rank of $D_{M} / M_{G}$.

Note that $M$ is a major subgroup of $G$, then $q_{G}(M) \neq 1$ if and only if $G / M_{G}$ is not in $\mathcal{U}^{*}$. Consequently the following result holds.

Corollary 2. Let $G$ be a group. Then $G \in \mathcal{U}^{*}$ if and only if the extended index of $M$ is 1 for every major subgroup $M$ of $G$.

Note that the above result extends a well known result of Huppert [8, VII, (2.2)]. Bathia [6] proved that the intersection of all maximal subgroups of a finite group of composite index is a supersoluble characteristic subgroup of the group. Our aim now is to obtain a similar result in our universe.

Definition 3. Let $G$ be a group. We define

$$
\begin{aligned}
L(G) & =\bigcap\left\{M: M \text { is a major subgroup of } G \text { such that } q_{G}(M) \neq 1\right\} \\
& =\bigcap\left\{M: M \text { is a major subgroup of } G \text { such that } G / M_{G} \notin \mathcal{U}^{*}\right\} .
\end{aligned}
$$

We stipulate that $L(G)=G$ if the above set of major subgroups is empty. 
Theorem 4. Let $G$ be a group. Then $L(G)$ is a characteristic $\mathcal{U}^{*}$-subgroup of $G$.

Proof: It is clear that $L(G)$ is a characteristic subgroup of $G$. We prove now that $L(G)$ belongs to $\mathcal{U}^{*}$. If the above set of major subgroups is empty then $L(G)=G \in \mathcal{U}^{*}$ by Corollary 1 . Then we may assume that this set is non-empty. Assume first that $\mu(G)=1$. Let $L(G)^{\mathcal{U}^{*}}$ be the $\mathcal{U}^{*}$-residual of $L(G)$, that is, the intersection of all normal subgroups $N$ of $L(G)$ such that $L(G) / N \in \mathcal{U}^{*}$. By Theorem 1, we have that $L(G) / L(G)^{\mathcal{U}^{*}}$ is a $\mathcal{U}^{*}$-group. We will show that $L(G)^{\mathcal{U}^{*}}$ is a subgroup of $\mu(G)$. Obviously $L(G)^{\mathcal{U}^{*}}$ is contained in every major subgroup $M$ of $G$ such that $G / M_{G} \notin \mathcal{U}^{*}$. Suppose now that $M$ is a major subgroup of $G$ such that $G / M_{G} \in \mathcal{U}^{*}$. Then, the $\mathcal{U}^{*}$-residual of $G, G^{\mathcal{U}^{*}}$, is contained in $M_{G}$. Since $\mathcal{U}^{*}$ is subgroup-closed, it follows that $L(G)^{\mathcal{U}^{*}} \leq G^{\mathcal{U}^{*}}$. Consequently, $L(G)^{\mathcal{U}^{*}}$ is a subgroup of $M$. This implies that $L(G)^{\mathcal{U}^{*}}$ is contained in every major subgroup of $G$ and hence $L(G)^{\mathcal{U}^{*}} \leq \mu(G)=1$. We conclude that $L(G) \in \mathcal{U}^{*}$.

Suppose now that $\mu(G) \neq 1$ and consider $G / \mu(G)$. By the above argument we have that $L(G / \mu(G)) \in \mathcal{U}^{*}$. Moreover, $L(G / \mu(G))=$ $L(G) / \mu(G)$. Therefore it follows from Theorem 3 that $L(G)$ is a $\mathcal{U}^{*}$-group.

It is well known that if $G$ is a finite supersoluble group, then $G$ has a normal Sylow $p^{\prime}$-subgroup [8, VII, (2.1)] for the smallest prime $p$ dividing the order of $G$. Moreover, the derived subgroup of $G$ is nilpotent. The corresponding versions in our universe for the class $\mathcal{U}^{*}$ are the following:

Theorem 5. Let $G$ be a $\mathcal{U}^{*}$-group. Then:

a) G has a normal Sylow $p^{\prime}$-subgroup for the smallest prime $p$ dividing the orders of the elements of $G$.

b) $G^{\prime} \leq F(G)$; in particular, $G^{\prime}$ belongs to $\mathfrak{B}$.

Proof:

a) Let $M$ be a major subgroup of $G$ and denote $M_{G}=\operatorname{Core}_{G}(M)$. Suppose that the result is true for $G / M_{G}$ for every major subgroup $M$ of $G$. Let $Q$ be a Sylow $p^{\prime}$-subgroup of $G$. Since all $p^{\prime}$-subgroups of $G$ are conjugate and $Q M_{G} / M_{G}$ is a Sylow $p^{\prime}$-subgroup of $G / M_{G}$ by [7], it follows that $G=\left(\mathrm{N}_{G}(Q)\right) M_{G}$ for every major subgroup $M$ of $G$. Since every proper subgroup of $G$ is contained in a major subgroup of $G$, it follows that $G=\mathrm{N}_{G}(Q)$. That is, $G$ contains a normal Sylow $p^{\prime}$-subgroup. Hence there is no loss of generality in assuming that $M_{G}=1$ and then $G$ is either a finite 
primitive soluble group or $G$ is a semiprimitive group by $[\mathbf{2}$, Theorem 1]. In the first case, $G$ is a finite supersoluble group and then the result is true [8, VII, (2.1)]. Consequently we may assume that $G$ is a semiprimitive group. Then $G=\left[G^{0}\right] M$, where $G^{0}$ is a divisibly irreducible $\mathbb{Z} G$-module, which is a $q$-group for some prime $q$, such that $\mathrm{C}_{G}\left(G^{0}\right)=G^{0}$ and $M$ is a finite soluble group. Since $G \in \mathcal{U}^{*}$, we have that $G^{0}$ is a quasicyclic $q$-group. Therefore $G / \mathrm{C}_{G}\left(G^{0}\right)=G / G^{0}$ is a subgroup of a cyclic group of order $q-1$ if $q \neq 2$ or a cyclic group of order 2 if $q=2$. Consequently $p$ divides $|M|$. If $q=2$ then $G$ is a 2-group and hence the Sylow $2^{\prime}$-subgroup of $G$ is trivial. Let assume that $q \neq 2$. In particular $p \neq q$. Now $M$ is cyclic and so $M$ has a normal Sylow $p^{\prime}$-subgroup, $R$ say. Then $Q=G^{0} R$ is a normal $p^{\prime}$-subgroup of $G$, which is our claim.

b) Let $H / K$ be a $\delta$-chief factor of $G$. In particular, $H / K$ is a $p$-group for some prime $p$. Since $G \in \mathcal{U}^{*}$ it follows that $G / \mathrm{C}_{G}(H / K)$ is in the class $\mathfrak{A}(p-1)$ if $p \neq 2$ or in the class $\mathfrak{A}(2)$ if $p=2$. Therefore $G^{\prime} \leq \mathrm{C}_{G}(H / K)$ for every $\delta$-chief factor $H / K$ of $G$. Since the Fitting subgroup $F(G)$ of a group $G$ is the intersection of the centralizers of all $\delta$-chief factors of $G$ by [3, Theorem 7], we conclude that $G^{\prime} \leq F(G)$. In particular $G^{\prime}$ belongs to $\mathfrak{B}$.

\section{References}

[1] R. Baer, Supersoluble groups, Proc. Amer. Math. Soc. 6 (1955), $16-32$.

[2] A. Ballester-Bolinches and S. Camp-Mora, A GaschützLubeseder type theorem in a class of locally finite groups, J. Algebra 221(2) (1999), 562-569.

[3] A. Ballester-Bolinches and T. Pedraza, On a class of generalized nilpotent groups, J. Algebra 248(1) (2002), 219-229.

[4] A. Ballester-Bolinches and T. Pedraza, The Fitting subgroup and some injectors of radical locally finite groups with min- $p$ for all p, Comm. Algebra 31(1) (2003), 483-492.

[5] A. Ballester-Bolinches and T. Pedraza, A local approach to a class of locally finite groups, Bull. Austral. Math. Soc. 68(1) (2003), 45-56.

[6] H. C. Bhatia, A generalized Frattini subgroup of a finite group, Ph. D. Thesis, Michigan State Univ., East Lansing (1972). 
[7] M. R. Dixon, "Sylow theory, formations and Fitting classes in locally finite groups", Series in Algebra 2, World Scientific Publishing Co., Inc., River Edge, NJ, 1994.

[8] K. Doerk And T. Hawkes, "Finite soluble groups", de Gruyter Expositions in Mathematics 4, Walter de Gruyter \& Co., Berlin, 1992.

[9] B. Hartley, A dual approach to Černikov modules, Math. Proc. Cambridge Philos. Soc. 82(2) (1977), 215-239.

[10] D. J. S. Robinson, "Finiteness conditions and generalized soluble groups", Part 1, Ergebnisse der Mathematik und ihrer Grenzgebiete 62, Springer-Verlag, New York-Berlin, 1972; Part 2, Ergebnisse der Mathematik und ihrer Grenzgebiete 63, Springer-Verlag, New York-Berlin, 1972.

[11] M. J. Tomkinson, A Frattini-like subgroup, Math. Proc. Cambridge Philos. Soc. 77 (1975), 247-257.

[12] M. J. Tomkinson, Finiteness conditions and a Frattini-like subgroup, in: "Proceedings of the Second International Group Theory Conference" (Bressanone, 1989), Rend. Circ. Mat. Palermo (2) Suppl. No. 23 (1990), 321-335.

[13] M. J. Tomkinson, Schunck classes and projectors in a class of locally finite groups, Proc. Edinburgh Math. Soc. (2) 38(3) (1995), $511-522$.

\author{
Adolfo Ballester-Bolinches: \\ Departament d'Àlgebra \\ Universitat de València \\ Dr. Moliner 50 \\ 46100 Burjassot, València \\ Spain \\ E-mail address: Adolfo.Ballester@uv.es \\ Tatiana Pedraza: \\ Departamento de Matemática Aplicada \\ Universidad Politécnica de Valencia \\ Escuela Politécnica Superior de Alcoy \\ 03801 Alcoy, Alicante \\ Spain \\ E-mail address: tapedraz@mat.upv.es
}

Primera versió rebuda el 2 de setembre de 2004, darrera versió rebuda el 20 de desembre de 2004. 